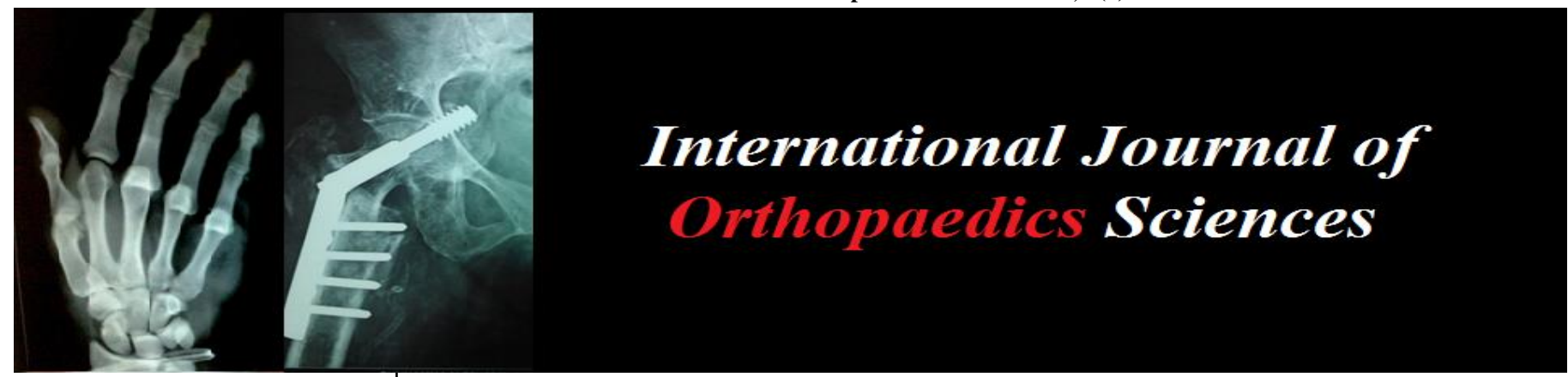

E-ISSN: 2395-1958

P-ISSN: 2706-6630

IJOS 2020; 6(3): 96-98

(C) 2020 IJOS

www.orthopaper.com

Received: 22-05-2020

Accepted: 24-06-2020

Dr. Hrishikesh Suresh Saodekar Associate Professor, Department of Orthopedics, Geetanjali Medical College and Hospital, Udaipur, Rajasthan, India

\section{Prevalence and clinical outcomes of spinal tuberculosis with multi drug resistance}

\section{Dr. Hrishikesh Suresh Saodekar}

DOI: https://doi.org/10.22271/ortho.2020.v6.i3b.2184

\begin{abstract}
Introduction: Due to lack of data for the early diagnosis and treatment of multi drug resistant spinal tuberculosis, the aim of the present study was to estimate the prevalence of multi drug resistant spinal tuberculosis and study the clinical outcomes.

Materials \& methods: A total of 80 patients were selected for the study. On the basis of the clinicoradiological diagnosis the anti-tubercular treatment was started in all the included patients diagnosed with drug resistant spinal tuberculosis. All the tissue specimen from the biopsy and samples obtained were subjected to histopathological examination, CBNAAT and MGIT culture.

Results: Functional and clinical outcome of the patients were evaluated by VAS and ASIA grading. The baseline score of the VAS score was found to be $6-7$, this score was used to compare with the follow up score. When VAS and ASIA score was compared with previous follow up visit VAS score, significant improvement was noted up to 9 months.

Conclusion: In conclusion, early detection of MDR Spinal Tuberculosis would be helpful to avoid consequences arising due to in appropriate chemotherapy and, hence, would also result in favourable outcomes. However, further similar studies with larger samples are needed before we can make definitive statement about the usage of CBNAAT Test as routine diagnostic tool in suspected MDR Spinal Tuberculosis cases.
\end{abstract}

Keywords: Multi drug resistant, tuberculosis, prevalence, CBNAAT

\section{Introduction}

The bacterial disease tuberculosis is spread through air from person to person. Major organs affected by tuberculosis are lungs. However, it can also affect the other part of body like brain, kidney or the spine ${ }^{[1]}$. Most of the cases tuberculosis is curable and treatable however if the timely proper treatment is not done than there is mortality of the person. Drug resistant tuberculosis occurs when the causative bacteria become resistant to the specific drug that is use to treat tuberculosis, that means that drug can not kill the bacteria causing tuberculosis ${ }^{[2,3]}$.

The spread of tuberculosis occur through air from one person to another person. The bacteria causing the tuberculosis are put in the air through sneezes, singing, speak or throat coughs of the infected one ${ }^{[4]}$. When the people nearby do breathe the bacteria in the air than can get infected. Total of 1 to $3 \%$ cases of tuberculosis affects the spine. It is the most common form affecting the vertebral bones and the intervertebral discs. Spinal tuberculosis with drug resistant is an emerging health problem in the developed and developing countries ${ }^{[5,6]}$.

In the developing countries there is alarming rise in the multi drug resistant tuberculosis. In the year 2016 around 30 countries were declared as high multi drug resistant tuberculosis burden countries. $^{7}$ A total of $6,00,000$ new cases of tuberculosis with Rifampicin resistant were notified in 2016 by WHO, out of which majority of cases where of multi drug resistant. Almost around half of the cases were from India, Russia and China. The percentage of patients in India with either MDR-TB or RR-TB is estimated to be $2.8 \%$ among new TB cases and $12 \%$ among previously treated cases ${ }^{[8]}$.

Drug resistant spinal tuberculosis posses a set of challenges when compared to drug resistant pulmonary tuberculosis. In the patients with pulmonary tuberculosis it is easy to perform drug sensitivity test, due to easy availability of the sputum sample ${ }^{[9,10]}$. However, there is difficult to obtain sample in spinal tuberculosis so the drug sensitivity test is difficult. Spinal tuberculosis is a pauci-bacillary type of diseases hence even if the adequate sample is obtained
Corresponding Author: Dr. Hrishikesh Suresh Saodekar Associate Professor, Department of Orthopedics, Geetanjali Medical College and Hospital, Udaipur, Rajasthan, India 
the probability of cultural sensitivity as well as bacterial growth is very low ${ }^{[11]}$ Also due to presence of spinal deformity and neurological complications the diagnosis of spinal tuberculosis is often delayed ${ }^{[12]}$. Hence the present study was done with the sole objective to estimate the prevalence of multi drug resistant spinal tuberculosis and study the clinical outcomes.

\section{Materials \& methods}

The present study is the hospital-based prevalence study. The present study was done in the department of orthopaedics, government medical college \& hospital. The study duration was of 3 years. All the patients were explained about the study. The ethical committee of the institute was informed about the study and the ethical clearance certificate was obtained. The patients diagnosed with the clincio-radiological diagnosis of spinal tuberculosis and those who were ready to participate in the study and provided with the written inform consent were included in the study. A total of 80 patients were selected for the study. All the patients who refuse to give the written consent and diagnosed with other malignancy through biopsy were excluded from the study.

All the patients subjected to the centre with suspected spinal tuberculosis were evaluated with CT scan and MRI. All patients in whom surgery was not indicated were subjected to percutaneous transpedicular on OPD basis. CT guided or the fluoroscopy guided biopsy was done under the local anaesthesia. Remaining patients with neurological deficit or with indication of surgery were admitted and the biopsy material was collected intra-operatively and sent for investigation.

On the basis of the clinico-radiological diagnosis the antitubercular treatment was started in all the included patients diagnosed with drug resistant spinal tuberculosis. All the tissue specimen from the biopsy and samples obtained were subjected to histopathological examination, CBNAAT and MGIT culture. Culture of those samples which showed rifampicin resistance on CBNAAT was followed up for DST (Drug Susceptibility Test) and the treatment accordingly modified if required. After the drug sensitivity report for 1stand 2nd line anti-tuberculosis drug was known in rifampicin resistance cases, individualized chemotherapy was formulated. All cases were followed up at 3-month, 6-month, 9 months. The data collected were analysed for statistical significance using SPSS.

\section{Results}

The present study was aimed to estimate the prevalence of multi drug resistance spinal tuberculosis and study the clinical outcomes. Total of 80 patients were included in the study. The age range of the patients include in the study was from 20 years to 70 years and the mean age of the patients included in the study was around 42 years. Out of total 80 patients included there were 50 males and 30 females.

The most common complain of the patients included was back pain in all the cases. The secondary symptoms were loss of weight, loss of appetite and evening rise in temperature seen in 56 cases and 46 cases had neurological deficit. Most commonly involved area is the thoracic area, followed by lumbosacral and lastly thoracolumber junction was involved. In most of the cases only two vertebras were affected (50 cases) and followed by three vertebrae in 18 cases and 12 lesion shows skip lesions in radiological evaluation.

In majority of the cases, the samples of the tissue were collected through fluoroscopy guided biopsy. In 42 cases the biopsy was done through guided technique. Intra-operatively tissue sampling was done in 34 cases and in 4 cases CT guided biopsy was done. Of the 42 cases the multi drug resistant tuberculosis was diagnosed in 24 cases. Of the 34 cases intra operatively sampling was done 22 cases were diagnosed with multidrug resistant tuberculosis. In both the CT guided biopsy cases they were found negative for MTB.

Total of 80 cases of suspected spinal tuberculosis in the present study, through CBNAAT the mycobacterium tuberculosis was detected in 46 patients. Of the 46 patients, 40 patients showed sensitivity to rifampicin and 6 patients were found to be resistant. Of the total cases, the histopathological signs of chronic granulomatous features of tuberculosis were seen in 36 cases and in rest of the 44 cases the histopathology was inconclusive.

Functional and clinical outcome of the patients were evaluated by VAS and ASIA grading. The baseline score of the VAS score was found to be $6-7$, this score was used to compare with the follow up score. When VAS score was compared with previous follow up visit VAS score, significant improvement was noted up to 9 months. Maximum improvement in VAS score occurred in first 6 months of follow up (50\%) (Table 1).

As per the ASIA grades, the maximum number of cases belong to grade $\mathrm{E}$ and minimum cases were in grade $\mathrm{D}$. At the follow up interval significant improvement was seen in all the cases. (Table 2)

\section{Discussion}

The major challenging part in the tuberculosis is the treatment of multi drug resistant tuberculosis. There is found significant rise in number of cases in the MDRTB in the developing countries. The causative factors of the multi drug resistance do vary and the possible causative agent is not known ${ }^{[13]}$. Various risk factors for developing the resistance to the drugs are young age, patients affected with human immunodeficiency virus infection, person in contact with person previously infected with multi drug resistant tuberculosis and had previous treatment of tuberculosis ${ }^{[14]}$.

Although there is no particular guideline for the treatment of drug resistant tuberculosis; the $\mathrm{WHO}$ has outlined specific recommendation for the treatment of multi drug resistant tuberculosis. All patients in this study were treated according to the WHO protocol for drug resistant pulmonary tuberculosis after drug susceptibility testing.

The diagnosis of the spinal tuberculosis is done on the clinic radiological findings that include MRI. Spinal tuberculosis is considered as deep seated paucibacillary lesions. However, the confirmation is done with the histological and bacteriological evaluation that requires the invasive procedure to obtain the tissue sample for the examination. Hence both the clinic radiological as well as histopathological examination is necessary for the confirmatory diagnosis of spinal drug resistant tuberculosis. In India, prevalence of primary MDR-TB in newly diagnosed cases has been observed to be 3.4 per cent or less. The prevalence is found to be at a low level in most of the country where it has been studied.

In the present study the biopsy with fluoroscopic guidance was done in 42 patients out of 80 patients. The procedure was performed under local anaesthesia. Biopsy with help of CT guided was seen in 4 cases and in 34 cases the samples were obtained intra operatively. With the help of CBNAAT mycobacterium tuberculosis was detected in 24 patients. Shrestha et al. ${ }^{[15]}$ in their study detected Mycobacterium 
tuberculosis in 19 patients from 23 cases. In 4 patients we have performed CT guided biopsy, but in both tissue samples MTB was not detected.

The neurological deficit was assessed with the help of American spinal injury association. In the follow up period the significant improvement was seen patients. The follow up was studied at interval of 3 months for the period of 9 months. This result was comparable to study conducted by Li et al. ${ }^{[16]}$ On retrospective analysis of management of drug resistant spinal tuberculosis in 35 patients, improvement was noted in ASIA scores, from 17 cases with Grade $\mathrm{E}$ at initial presentation to 29 Cases with Grade $\mathrm{E}$ at the final follow up visit.

The VAS score was used to assess the pain with the baseline score of 6 to 7. In the follow up period the improvement was found in the VAS score for the pain in the back ache. The improvement in the VAS score was found to be consistently increased in the follow up period of 9 months. Maximum improvement was seen in first six months as per the VAS score. Qing-Yi et al. ${ }^{[17]}$ reported similar improvement in VAS score in their study of treatment effect and postoperative complication in 54 patients of thoracic and lumbar spinal tuberculosis.5 In their study VAS score of $7.8 \pm 1.7$ improved to $3.2 \pm 2.1$ at the last follow up.

\section{Conclusion}

In conclusion, early detection of MDR Spinal Tuberculosis would be helpful to avoid consequences arising due to in appropriate chemotherapy and, hence, would also result in favourable outcomes. However, further similar studies with larger samples are needed before we can make definitive statement about the usage of CBNAAT Test as routine diagnostic tool in suspected MDR Spinal Tuberculosis cases.

Table 1: Comparison of base line score with 3 months interval analogue scale

\begin{tabular}{|c|c|c|c|c|c|}
\hline VAS & F/u & N & Mead & SD & P value \\
\hline Pair 1 & Baseline & 80 & 6.12 & 0.62 & $<0.01$ \\
\hline & $3 \mathrm{~m}$ & 80 & 5.67 & 1.23 & $<0.01$ \\
\hline Pair 2 & $3 \mathrm{~m}$ & 80 & 5.67 & 1.23 & $<0.01$ \\
\hline & $6 \mathrm{~m}$ & 80 & 4.10 & 1.10 & $<0.01$ \\
\hline Pair 3 & $6 \mathrm{~m}$ & 44 & 3.49 & 1.04 & $<0.01$ \\
\hline & $9 \mathrm{~m}$ & 44 & 2.10 & 1.40 & $<0.01$ \\
\hline
\end{tabular}

Table 2: Progression of ASIA grading over periodic follow up

\begin{tabular}{|c|c|c|c|c|c|}
\hline ASIA & A & B & C & D & E \\
\hline Baseline & 106 & 16 & 10 & 8 & 34 \\
\hline 3 months & 0 & 14 & 14 & 6 & 38 \\
\hline 6 months & 0 & 4 & 16 & 14 & 44 \\
\hline 9 months & 0 & 0 & 2 & 10 & 32 \\
\hline
\end{tabular}

\section{References}

1. Burrill J, Williams CJ, Bain G, Conder G, Hine AL, Misra RR. Tuberculosis: a radiologic review. Radiographics. 2007; 27:1255-73.

2. Zhang Y, Post-Martens K, Denkin S. New drug candidates and therapeutic targets for tuberculosis therapy. Drug discovery today. 2006; 11:21-7.

3. Dheda K, Gumbo T, Maartens G, Dooley KE, McNerney $\mathrm{R}$, Murray $\mathrm{M}$ et al. The epidemiology, pathogenesis, transmission, diagnosis, and management of multidrugresistant, extensively drug-resistant, and incurable tuberculosis. The lancet Respiratory medicine. 2017; 5:291-360.

4. CDC A. Diagnostic standards and classification of tuberculosis. American Review of Respiratory Disease, 1990, 142.

5. Harisinghani MG, McLoud TC, Shepard J-AO, Ko JP, Shroff MM, Mueller PR. Tuberculosis from Head to Toe 1: (CME available in print version and on RSNA Link). Radiographics. 2000; 20:449-70.

6. Tyagi R. Spinal infections in children: a review. Journal of orthopaedics. 2016; 13:254-8.

7. Javed H, Tahir Z, Hashmi HJ, Jamil N. A cross-sectional study about knowledge and attitudes toward multidrugresistant and extensively drug-resistant tuberculosis in a high-burden drug-resistant country. International journal of mycobacteriology. 2016; 5:128-34.

8. Shah I, Poojari V, Meshram H. Multi-Drug Resistant and Extensively-Drug Resistant Tuberculosis. The Indian Journal of Pediatrics, 2020, 1-7.

9. Koul A, Arnoult E, Lounis N, Guillemont J, Andries K. The challenge of new drug discovery for tuberculosis. Nature. 2011; 469:483-90.

10. Dheda K, Gumbo T, Gandhi NR, Murray M, Theron G, Udwadia $\mathrm{Z}$ et al. Global control of tuberculosis: from extensively drug-resistant to untreatable tuberculosis. The lancet Respiratory medicine. 2014; 2:321-38.

11. Gautam M, Karki P, Rijal S, Singh R. Pott's spine and paraplegia. Journal of the Nepal Medical Association, $2005,44$.

12. Jain AK, Jaggi KR, Bhayana H, Saha R. Drug-resistant spinal tuberculosis. Indian Journal of Orthopaedics. 2018; 52:100-7.

13. Caminero J. Multidrug-resistant tuberculosis: epidemiology, risk factors and case finding [State of the art series. Drug-resistant tuberculosis. Edited by CY. Chiang. Number 4 in the series]. The International Journal of Tuberculosis and Lung Disease. 2010; 14:38290.

14. Sharma S, Mohan A. Multidrug-resistant tuberculosis. Indian Journal of Medical Research. 2004; 120:354-76.

15. Shrestha D, Shrestha R, Dhoju D. Fluoroscopy guided percutaneous transpedicular biopsy for thoracic and lumbar vertebral body lesion: technique and safety in 23 consecutive cases. Kathmandu University Medical Journal. 2015; 13:256-60.

16. Li L, Zhang Z, Luo F, Xu J, Cheng $\mathrm{P}, \mathrm{Wu} \mathrm{Z}$ et al. Management of drug-resistant spinal tuberculosis with a combination of surgery and individualised chemotherapy: a retrospective analysis of thirty-five patients. International orthopaedics. 2012; 36:277-83.

17. He Q-Y, Xu J-Z, Zhou Q, Luo F, Hou T, Zhang Z. Treatment effect, postoperative complications, and their reasons in juvenile thoracic and lumbar spinal tuberculosis surgery. Journal of orthopaedic surgery and research. 2015; 10:156. 